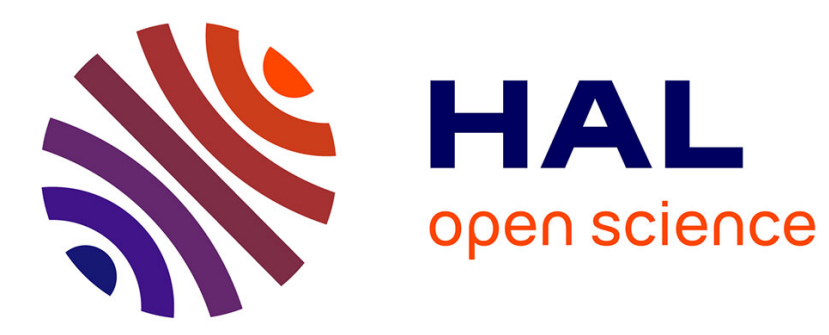

\title{
La production doctorale de la doctrine juridique : division du travail académique et politisation des thèses en droit sur les modes de scrutin (1885-1923)
}

Thomas Marty

\section{- To cite this version:}

Thomas Marty. La production doctorale de la doctrine juridique : division du travail académique et politisation des thèses en droit sur les modes de scrutin (1885-1923). Revue d'histoire des sciences humaines, 2011, 25 (2), p 281 - 304. 10.3917/rhsh.025.0265 . hal-01366604

\section{HAL Id: hal-01366604 https://hal.science/hal-01366604}

Submitted on 14 Sep 2016

HAL is a multi-disciplinary open access archive for the deposit and dissemination of scientific research documents, whether they are published or not. The documents may come from teaching and research institutions in France or abroad, or from public or private research centers.
L'archive ouverte pluridisciplinaire HAL, est destinée au dépôt et à la diffusion de documents scientifiques de niveau recherche, publiés ou non, émanant des établissements d'enseignement et de recherche français ou étrangers, des laboratoires publics ou privés. 


\section{LA PRODUCTION DOCTORALE DE LA DOCTRINE JURIDIQUE : DIVISION DU TRAVAIL ACADÉMIQUE ET POLITISATION DES THĖSES EN DROIT SUR LES MODES DE SCRUTIN (1885-1923) Thomas Marty}

Ed. Sc. Humaines | « Revue d'Histoire des Sciences Humaines »

$2011 / 2 n^{\circ} 25$ | pages 265 à 291

ISSN 1622-468X

ISBN 9782361060237

Article disponible en ligne à l'adresse :

http://www.cairn.info/revue-histoire-des-sciences-humaines-2011-2-page-265.htm

\section{Pour citer cet article :}

Thomas Marty, « La production doctorale de la doctrine juridique : division du travail académique et politisation des thèses en droit sur les modes de scrutin (1885-1923) », Revue d'Histoire des Sciences Humaines 2011/2 (n²5), p. 265-291. DOI 10.3917/rhsh.025.0265

Distribution électronique Cairn.info pour Ed. Sc. Humaines.

(C) Ed. Sc. Humaines. Tous droits réservés pour tous pays.

La reproduction ou représentation de cet article, notamment par photocopie, n'est autorisée que dans les limites des conditions générales d'utilisation du site ou, le cas échéant, des conditions générales de la licence souscrite par votre établissement. Toute autre reproduction ou représentation, en tout ou partie, sous quelque forme et de quelque manière que ce soit, est interdite sauf accord préalable et écrit de l'éditeur, en dehors des cas prévus par la législation en vigueur en France. Il est précisé que son stockage dans une base de données est également interdit. 


\title{
La production doctorale de la doctrine juridique : division du travail académique et politisation des thèses en droit sur les modes de scrutin (1885-1923)
}

\author{
Thomas MarTy
}

\begin{abstract}
Résumé
Les thèses de droit soutenues entre 1885 et 1923 sur la représentation proportionnelle comme mode de scrutin des élections législatives forment un ensemble de connaissances qui permet de juger la part prise par le droit constitutionnel dans une controverse tout autant académique que politique. Les professeurs de droit constitutionnel, tant à Paris qu'en province, délaissent cette expertise malgré quelques entreprises militantes éparses (SAlEILlEs, Villey, etc.). Leurs étudiants formulent alors un savoir académique vite enserré par les contraintes des compositions récurrentes des jurys de thèse. La politisation rampante de ces thèses s'exprime de trois manières : un premier ensemble de thèses tente de rechercher dans les origines juridiques et philosophiques de la représentation proportionnelle des garanties supplémentaires de son opportunité politique ; d'autres thèses relatent des exemples étrangers d'application de la représentation proportionnelle et en font des modèles à suivre pour la France ; enfin, ces travaux se calquent la plupart du temps sur la chronologie et les contingences proprement parlementaires de la question.
\end{abstract}

Mots-clés : Faculté de droit - Étudiants en thèse - Études électorales - Réforme électorale - Troisième République.

\section{Abstract : The Doctoral Contribution to Juridical Thought. Academic Working and} Political Interests in Ph. D Thesis about Electoral Systems (1885-1923)

Between 1885 and 1923, Ph. D thesis on proportional representation (PR) and electoral reform have been main constitutional corpus to follow this intellectual and political controversy. In Paris and in Province, professors desert this part of doctrine in spite of some militant activities (Saleilles, Villey). Students had produced their thesis under constraint because the boards of examiners were always the same. Firstly, some of these thesis have tried to find in philosophical and juridical aspects of PR the justification in order to introduce it in electoral law. In other thesis, the PR was justified simply because some european countries had used this electoral system. Last type of these printed thesis are bound by parliamentary calendar of electoral reform.

\footnotetext{
${ }^{1}$ Ce travail est issu d'une thèse de science politique en voie d'achèvement : « Le militantisme républicain et la statistique électorale au service de l'établissement de la représentation proportionnelle », Paris X. dir. Bernard Lacroix. Il a fait l'objet d'une première présentation lors du congrès de l'Association française de droit constitutionnel à Montpellier en 2005. Je remercie les organisateurs, M. Troper et A. Viala, ainsi que les commentateurs, A. Laquièze et B. Lacroix.
} 
Revue d'Histoire des Sciences Humaines

In conclusion, the political interests and the scientific knowledge seem to be also important in study of electoral systems.

Key-words : Faculty of law - Ph. D Students - Electoral studies - Electoral reformThird Republic. 
En France, l'expertise constitutionnelle s'est toujours délicatement positionnée par rapport au mode de scrutin devant régir les élections législatives ${ }^{2}$. Ses réticences concernant le système majoritaire, pratiqué tant sous la $\mathrm{III}^{\mathrm{e}}$ que la $\mathrm{V}^{\mathrm{e}}$ République, ont alimenté de vives critiques et quelques projets de réforme. Ce type de critiques subsiste encore dans la doctrine ${ }^{3}$; les projets de réforme éventuellement portés par les constitutionnalistes ne résistent guère aux contraintes politiques comme en témoigne le sort réservé au mode de scrutin dans le traitement législatif des propositions du comité Balladur de réforme des institutions à la fin de l'année $2007^{4}$. Il faut donc en revenir à des configurations passées qui pourraient expliquer ce constat par des raisons tout autant politiques qu'académiques. Plus particulièrement, la représentation proportionnelle comme mode de scrutin à adopter pour les élections législatives se présente à la jonction des $\mathrm{XIX}^{\mathrm{e}}$ et $\mathrm{XX}^{\mathrm{e}}$ siècles, comme un produit politique double : à la fois étendard de ceux pour qui la réforme électorale doit désormais embrasser l'activité inter-partisane (après avoir codifié les pratiques de vote elles-mêmes) ${ }^{5}$ mais aussi objet d'une controverse para-scientifique multiple dont le droit constitutionnel ne sera d'ailleurs qu'un élément parmi d'autres. L'espace académique juridique s'avère un point d'observation privilégié de ce double mouvement. Certaines productions constitutionnelles prétendent en effet avoir leur mot à dire dans cette entreprise de réforme. Tout au long d'une controverse qui débute en 1885 avec l'expérience laconique du scrutin de liste et se termine provisoirement en 1919 avec l'adoption partielle de la représentation proportionnelle, les thèses de droit soutenues sur ce sujet forment un corps de connaissance extrêmement fourni et varié permettant de suivre la chronologie et les aléas de ce processus politico-législatif.

On se propose d'inventorier les conditions de production et de réception de 23 thèses de droit public (ou d'économie politique) portant sur la représentation proportionnelle ou les modes de scrutin en général (cf. tableau 1). Il est nécessaire de revenir au préalable sur les positions engendrées par ce débat public sur les règles électorales depuis les débuts de la III $^{\mathrm{e}}$ République en remarquant par exemple la place faite initialement au savoir juridique dans des controverses institutionnelles plus larges. On observera la relative marginalité du droit constitutionnel, par exemple le faible nombre de thèses soutenues à ce propos avant 1900, puis progressivement la contribution propre des travaux de thèse à la définition d'une dichotomie entre le "système " majoritaire existant - décrit avec le langage de la critique historique et politique, parfois partisane - et le souhait d'un «principe " proportionnel abordé quant à lui à l'aune des théories juridiques de la souveraineté. L'engagement, parfois militant, des professeurs de droit constitutionnel à propos de la représentation proportionnelle tout au long de cette période permet en outre de mesurer les contraintes et les ressources que le choix de ce sujet de fin d'études pouvait alors comporter. Il nous faudra reconsidérer les réseaux et les raisons académiques, visibles à travers la composition des jurys, qui structurent ce travail doctoral en instituant des conditions intellectuelles et politiques plus ou moins

\footnotetext{
${ }^{2}$ Il n'existe dans la production française contemporaine sur les modes de scrutin que peu de synthèses rédigées par des constitutionnalistes, cf. toutefois PAUVERT, 2006.

${ }^{3}$ Susani, 2007, 145-159; AROMATORIo, 2007, 601-622.

${ }^{4}$ Pauvert, 2007.

${ }^{5}$ Cf. Garrigou, 2002 et Déloye et Ihl, 2008.
} 
favorables au choix d'un tel sujet ${ }^{6}$. Il sera ensuite nécessaire d'aborder les dynamiques d'écriture et de politisation qui composent ces travaux et finissent de convaincre du rôle primordial joué par la thèse $\mathrm{e}^{7}$ dans la publicisation d'une cause politique dont la mise en forme intellectuelle juridique détermine en partie la légitimité.

\section{La réforme électorale : des origines politiques à la codification intellectuelle}

Entre la « défaite de Sedan » et le début de la Commune de Paris, des élections ont lieu en février 1871 sans qu'aucune nouvelle loi électorale ne soit votée. Les premières élections de la $\mathrm{III}^{\mathrm{e}}$ République sont donc pratiquées sous le régime de la loi de 1849 (un scrutin de liste départemental) quelque peu aménagé à la marge par un décret. Il s'ouvre alors une période d'incertitude en matière de loi électorale qui ne sera solutionnée qu'en 1889 , permettant seulement à partir de cette date l'établissement d'une véritable controverse doctrinale. Il n'y a donc pas jusqu'à la réalité des textes applicables en matière de mode de scrutin législatif qui n'ait pu influer sur la production doctrinale représentée par un certain nombre d'écrits au nombre desquels les thèses de droit ne comptent pas dans ces premières décennies de la République (pas plus, d'ailleurs, que les manuels et cours des professeurs qui les dirigent). Le droit se montre absent de toute expertise ou bien se situe en porte-à-faux du fait même de la position péremptoire de certains de ses représentants. Le titulaire de la chaire de droit administratif parisienne, Anselme Batbie, également député et rapporteur monarchiste de la deuxième commission des Trente, est ainsi l'un de ceux qui introduisent le scrutin individuel tant à l'Assemblée Nationale que dans ses écrits antérieurs sous l'Empire : « la loi actuelle a formellement repoussé le scrutin de liste (...) On a voulu que chacun votât (...) pour des noms connus de lui (... $)^{8}$. Il n'est ainsi pas anodin que ce soit un spécialiste de droit administratif qui soit mis en première ligne. L'autonomie du droit constitutionnel n'étant pas encore conquise, les lois électorales qui se succèdent se feront jusqu'au début du $\mathrm{xx}^{\mathrm{e}}$ siècle sans expertise spécifique préalable. Les thèses de droit permettent de suivre cette mutation puisqu'elles ne sont que trois à être soutenues avant 1900 sur le corpus de 23 déterminé ci-après.

Dans un premier temps, les crises gouvernementales de la République naissante ${ }^{9}$ vont circonscrire le débat sur le mode de scrutin à une lutte quasi exclusivement politique qui exclut à peu près tout redoublement intellectuel de la controverse. La loi de 1875 n'est pas le fruit d'un consensus mais d'une transaction entre des positions a priori irréconciliables. Elle adopte certes le scrutin uninominal majoritaire à deux tours mais on sait que dès le départ des républicains de gauche comme L. Gambetta par exemple, étaient favorables au scrutin de liste. La loi du 24 mars 1885 reprend les principes fondamentaux de cette dernière modalité : candidatures de liste à l'échelon départemental, répartition majoritaire des sièges. Elle doit aux conditions

\footnotetext{
${ }^{6}$ Une approche relationnelle des jurys de thèse, avec de toutes autres méthodes et dans un tout autre contexte historique et disciplinaire, a été esquissée par GODECHOT et MARIOT, 2004.

${ }^{7}$ Voir les pistes de réflexion évoquées par NoIRIEL, 1991.

${ }^{8}$ BAtBie, 1861.

${ }^{9}$ Marty, 2010.
} 
conjoncturelles inattendues où elle trouvera à s'appliquer (l'épisode boulangiste) les raisons de son abandon dès les élections suivantes en 1889. La loi électorale de 1889 ne sera alors qu'une reprise du système de 1875. Adoptée sans réel débat public, la loi rétablissant le scrutin uninominal majoritaire à deux tours perdure jusqu'en 1919 en suscitant progressivement des critiques innovantes, tantôt théoriques (thèses $\mathrm{n}^{\circ} 3$ et 4 en 1897 et 1899) tantôt plus pratiques c'est-à-dire se contentant de reproduire les arguments échangés entre les acteurs politiques eux-mêmes (thèses $\mathrm{n}^{\circ} 10, \mathrm{n}^{\circ} 14, \mathrm{n}^{\circ} 15$, $\mathrm{n}^{\circ} 17$ en 1907, 1910, 1915 ainsi que la dernière thèse du corpus soutenue en 1923 et venant faire le bilan immédiat de la loi votée en 1919).

Cette loi électorale du 12 juillet 1919 introduit un mode de scrutin mixte (majorité absolue puis secondairement une répartition proportionnelle) qui ne sera appliqué qu'aux élections de 1919 et 1924 en produisant deux majorités opposées : le Bloc National, largement orienté à droite, puis le « Cartel des Gauches ». Cette instabilité dans les résultats, qui rompt avec les lentes évolutions de majorité avant la guerre, condamnera cette loi électorale à être supprimée. Le processus parlementaire de la réforme électorale avait été engagé dès le lendemain des élections de 1889 : pas moins de 53 propositions de loi proposèrent de réformer le mode de scrutin jusqu'en 1919 (dont plus de la moitié pour introduire le scrutin de liste proportionnel), sans compter les rapports et débats parlementaires, le tout formant la matière textuelle et intellectuelle première des thèses dont on entend ici comprendre l'influence sur la mise en forme du problème public de la réforme des règles du jeu électoral. Cette institutionnalisation de l'élection donne en effet sa forme la plus évidente à la mobilisation en faveur de la représentation proportionnelle. Parce qu'elle enrôle au-delà des cénacles d'élus et devient l'enjeu de la délimitation du groupe de ceux qui aspirent à entrer en politique ou à modéliser cette activité, la " représentation proportionnelle » (ou la " réforme électorale ») contribue à renforcer l'emprise des processus électoraux qu'elle dénonce. $\mathrm{Au}$ principe majoritaire elle oppose un affinement de cette majorité rendu possible par l'éclaircissement des programmes et des alliances, aux minorités délaissées par le scrutin uninominal abrupt elle oppose d'autres minorités.

Les différentes thèses " généralistes " du corpus ne manquent pas de contribuer à cette entreprise de classification des modes de scrutin. Ainsi, alors même que l'objet de sa thèse est le scrutin de liste, F. Sainz $\left(\mathrm{n}^{\circ} 13\right)$ distingue clairement en deux parties les manières dont le système majoritaire et le système proportionnel peuvent appliquer cette modalité de présentation des candidatures. Il va même jusqu'à construire toute son argumentation dans une perspective évolutionniste : le système majoritaire ne pouvant accueillir le scrutin de liste puisqu'il associe le vote de l'électeur à la personne candidate et non à l'idée défendue, seule la représentation proportionnelle est à même d'assumer cette fonction où l'électeur ne devra plus élire une personnalité mais plusieurs individus incarnant les mêmes idéaux.

Cette vision évolutionniste est accentuée tout au long de la thèse de G. Jaigu $\left(\mathrm{n}^{\circ} 17\right)$ : le scrutin majoritaire est affublé de trois défauts majeurs ${ }^{10}$ (l'inégalité des circonscriptions, l'abstentionnisme, l'omnipotence du second tour de scrutin) auxquels la représentation proportionnelle prétend justement remédier en innovant : le scrutin de liste (qui écrase

${ }^{10} \mathrm{~J}$ AIGU, 1911. 
les inégalités des circonscriptions en se situant au niveau départemental), le quotient électoral (qui accroît l'utilité du vote en annonçant le nombre de voix nécessaire à l'obtention d'un siège), l'utilisation des restes et l'apparentement (qui permettent de concentrer l'élection en un seul tour). L'étude des conséquences pratiques de ces solutions techniques constitue un espace beaucoup plus ouvert. La prospection propre à l'expertise proportionnelle concerne d'abord, avant même toutes les études spécifiques sur des cas étrangers ou sur la France, certaines thèses généralistes qui en synthétisent les « avantages » et les « inconvénients ». Deux thèses tout à fait opposées quant à leur position vis-à-vis de la représentation proportionnelle comme celles de $\mathrm{G}$. Bonnefoy $\left(\mathrm{n}^{\circ} 7\right)$ - hostile à la représentation proportionnelle - et de $\mathrm{M}$. Toupagnanine $\left(\mathrm{n}^{\circ} 21\right)$ - favorable à la représentation proportionnelle - se mettent ainsi d'accord sur les enjeux qu'il faut mettre en débat : l'égalité électorale postulée, le rôle des comités électoraux ou des partis et enfin le perfectionnement du parlementarisme semblent de ce point de vue trois dimensions fondamentales que les thèses sur les cas étrangers étudieront avec plus de précision.

Le principe même d'une séparation stricte entre scrutin majoritaire et scrutin proportionnel alimente donc la production originelle des thèses de droit en la matière. Loin de n'être que le reflet d'une situation politique indécise sur les réformes à entreprendre en la matière, le fondement de ce débat est également lié aux engagements publics qu'un certain nombre de professeurs de droit consentent à partir de la fin du XIX siècle. L'intérêt militant des professeurs de droit pour la représentation proportionnelle n'apparaît clairement que vers 1889 au moment donc où le mode de scrutin uninominal majoritaire à deux tours est durablement rétabli. En effet, l'introduction du scrutin de liste pour les élections législatives de 1885 ayant été jugée peu satisfaisante, le retour au scrutin d'arrondissement est voté pour les élections de 1889 après un embryon de débat contradictoire où la représentation proportionnelle apparaît en tant que telle comme argument légitime. C'est à ce moment-là qu'un mouvement se structure en France : la Société pour l'Étude de la Représentation Proportionnelle est fondée sous la tutelle de la Société de Législation Comparée et de personnalités proches de l'École Libre des Sciences Politiques (Georges Picot, Anatole Leroy-Beaulieu, Émile Boutmy) ${ }^{11}$. Bien que ne se composant donc pas principalement de membres de la Faculté de droit de Paris (hormis Bufnoir ${ }^{12}$ et Lyon-Caen), la Société envisage surtout la représentation proportionnelle à travers une approche comparée des différentes législations et/ou débats parlementaires à travers le monde. Toujours est-il que ce militantisme intellectuel ne va guère plus loin que la publication d'un volume qui synthétise cette expertise juridique ${ }^{13}$.

La seule autre mobilisation collective d'ampleur impliquant plus explicitement les juristes aura lieu en 1900 au moment de l'exposition universelle et du Congrès international de Droit Comparé14. Toujours sous l'impulsion de la Société de Législation Comparée, mais plus encore sous celle de Raymond Saleilles, une table ronde de droit public est organisée dont le thème central est la représentation proportionnelle. Fruit de

${ }^{11}$ Sur ces figures, cf. FAVRE, 1989.

${ }^{12}$ Claude Bufnorr a contribué à fonder la Société de législation comparée de laquelle émane cette Société pour l'étude de la RP. Il fût candidat, vaincu, aux élections législatives de 1893, postérieurement à cet engagement donc (НАKIM, 2007, 143-145).

${ }^{13}$ Vernes, 1888.

${ }^{14}$ Exposition internationale universelle, 1905. 
l'entrepreneur académique et politique qu'est Saleilles ${ }^{15}$, à la fois gendre de Bufnoir et auteur du principal article théorique sur la représentation proportionnelle dans les années $1890^{16}$, cette table ronde s'apparente plus à un exercice d'ingénierie constitutionnelle qu'à une véritable réunion d'expertise scientifique ${ }^{17}$. Elle met surtout en avant la centralité et la nouveauté de l'investissement spécifique des professeurs de droit et de leurs docteurs : Nicolas Saripolos, auteur un an auparavant d'une thèse monumentale sur la question à la faculté de droit de Paris, est invité à la table ronde dont l'instigateur R. Saleilles était membre de son jury ; Achille Mestre, agrégé de droit public à la faculté de Droit de Lille, qui avait rédigé un long compte rendu de la thèse de Saripolos pour la Revue Générale du Droit $^{18}$ est quant à lui chargé du rapport de synthèse.

Plus tardivement, seuls certains travaux de Joseph Barthélémy viendront rompre cet isolement ${ }^{19}$. Pourtant, toute analyse critique du parlementarisme à l'époque comporte de la part des professeurs de droit constitutionnel une évocation de la représentation proportionnelle ${ }^{20}$. Tous plus ou moins favorables à la mesure, ils s'appuient alors essentiellement sur une littérature militante (on pense aux travaux de G. Lachapelle et PG. La Chesnais ${ }^{21}$, aux travaux étrangers) et/ou parlementaire (on pense aux rapports élaborés en 1905 par le député de la Fédération Républicaine C. Benoist ou à partir de 1911 par le socialiste A. Groussier). L'agenda de la doctrine juridique se mêlant à l'évolution politique de la controverse sur le changement du mode de scrutin, elle va très vite devoir produire une expertise capable de suivre et même de précéder les aléas de ce débat politique. La division du travail professeurs/étudiants et les contraintes qui pèsent alors sur ces derniers lorsqu'ils s'engagent dans des thèses consacrées à la représentation proportionnelle, et plus généralement à la « réforme électorale », semblent donc claires : réfléchissant sur un droit des modes de scrutin qui n'existe quasiment pas et qui est déserté par les professeurs eux-mêmes ils sont investis de la mission de le fonder dans les voies suggérées par la position marginale de la doctrine elle-même. La place de la représentation proportionnelle dans le champ du débat doctrinal est donc dépendante de cette histoire plus académique qu'intellectuelle. L'espace des thèses ainsi produites apparaît bien comme le lieu central de cette histoire. Il l'est d'autant plus que la conjoncture universitaire connaît alors des évolutions majeures qui accréditent l'idée d'un poids accru de la thèse.

${ }^{15}$ Halpérin, 2007. Catholique convaincu, Saleilles s'affirme républicain après 1900 et devient proche de l'Abbé Lemire, député qui, à cette époque du moins, défend au parlement l'introduction de la représentation proportionnelle.

${ }^{16}$ Saleilles, 1898. Cet article est en fait un compte-rendu de la thèse d'A. Besson. Sur cet article, cf. l'interprétation de WALLON-LEDUCQ, 2004, 293-307.

${ }^{17}$ SACRiste, 2002, tome 2, 496.

${ }^{18}$ Mestre, 1899. Notons que dans le même numéro, N. Saripolos est invité à établir un compte rendu de la thèse d'A. Mestre (p 352-359). R. Saleilles avait lui aussi réalisé un compte rendu de la thèse de Saripolos (SAleilles, 1899).

${ }^{19}$ Barthélémy, 1912. Sur ces aspects de la pensée de Barthélémy, cf. SAULnier, 1996, 523-541. Les publications et activités politiques postérieures de Barthélémy (député et président de la Commission du suffrage universel) n'entrent pas ici en ligne de compte malgré le fil dessiné par une critique de la démocratie parlementaire sans doute inaugurée par l'ouvrage de 1912 et continuée progressivement par la suite (cf. BARTHÉLÉMY, 1918, 1928 et 1931).

${ }^{20}$ Voir les remarques rassemblées par REDOR, 1992, 111-114.

${ }^{21}$ La Chesnais, 1902 ; Lachapelle, 1911. 
Premièrement, les thèses deviennent des références nécessaires que les professeurs se sentent légitimes à citer dans leurs manuels, leurs monographies et leurs cours ${ }^{22}$. Léon Duguit par exemple, qui ne participe pourtant à aucun jury en matière de mode de scrutin, cite quatre thèses dans son Traité couvrant ainsi une large période de production entre 1887 et $1921^{23}$. Deuxièmement, si cette accumulation spécifique d'un capital scientifique vient à point nommé dans un univers concurrentiel, ce n'est qu'au prix d'une réévaluation de la place du doctorat en droit comme diplôme (et comme examen) et du changement ainsi opéré dans l'économie du travail professoral. Dès 1882, un décret permettait aux candidats à l'obtention du doctorat de choisir librement le sujet de leur deuxième dissertation (la première étant réservée au droit romain ${ }^{24}$. La réforme du doctorat en 1895 allait concéder la séparation des filières de droit privé et de droit public permettant ainsi l'émergence de nouvelles disciplines au sein des facultés de droit comme l'économie politique ou même la science politique ${ }^{25}$. Si on ajoute à cela la croissance du nombre de thèses et la difficulté qui s'ensuit de pouvoir les encadrer de manière convenable ${ }^{26}$, on comprendra aisément que l'appel d'air ait pu favoriser, si ce n'est l'innovation, du moins la diversification des sources et des méthodes de travail sur un objet de recherche non encore codifié par la doctrine. Pour la période qui nous intéresse ici, on dénombre ainsi une évolution assez nette : de 114 thèses de doctorat en droit soutenues en 1894 on passe à près de 600 en $1910^{27}$ (à Paris, le droit public couvre 95 des 353 thèses soutenues en $1910^{28}$ ). On s'aperçoit alors de la dévaluation relative du corpus de 23 thèses retenu dans notre étude : si elles sont principalement produites après 1900 c'est peut-être tout autant par une forme de suivisme politique qu'en relation avec l'appel d'air provoqué par les réformes mêmes du doctorat. Si le cursus permet en outre de se soustraire au service militaire, le prestige du diplôme s'en trouve affaibli. Il l'est d'autant plus qu'il sert alors à d'autres choses que l'orientation vers une carrière académique. En effet, la démocratisation du public étudiant des facultés de droit conduit à ce qu'une frange émergente des classes moyennes s'oriente vers des études de droit, et notamment le doctorat, afin de répondre aux opportunités de l'accroissement des débouchés (de nombreux journalistes ou avocats obtiennent ainsi un doctorat). C'est enfin l'ensemble des stratégies de publication des juristes qui favorise paradoxalement le développement de cette littérature doctorale.

\section{Les thèses sur la représentation proportionnelle et la composition de leurs jurys}

La déconsidération de "l'œuvre écrite » en tant que telle chez les juristes établis au profit de traités, manuels, notes de jurisprudence ou notes bibliographiques ${ }^{29}$ ainsi

\footnotetext{
${ }^{22}$ SACRISTE, 2002, tome 1, 265-270.

${ }^{23}$ Duguit, 1928, 726-753.

${ }^{24}$ Sur l'histoire du doctorat en droit, cf. IMBERT, 1984.

${ }^{25}$ Karady, 1986, 342.

${ }^{26}$ Charle, 1994.

${ }^{27}$ Moulinier, 2001, 109.

${ }^{28}$ IMBERT, 1984, 25.

${ }^{29}$ Charle, 1994, 253.
} 
que l'aspect repoussoir pour l'innovation intellectuelle que revêt l'agrégation et le peu de transformation de chaires vacantes donneraient aux thèses de doctorat une certaine forme d'unité. L'examen du respect de cette dernière hypothèse nous servira ici de guide. Le corpus de 23 thèses sélectionné est-il révélateur d'un savoir appliqué aux modes de scrutin qui serait spécifique à la science juridique ? A-t-il pu se transformer en capital d'expertise utile à ce qu'il prétendait défendre, dans son ensemble, c'està-dire l'introduction de la représentation proportionnelle comme mode d'accès aux fonctions législatives ? Au-delà de toute prise de position par rapport à la représentation proportionnelle et au-delà même de toute évocation de la concurrence académique, une ligne de clivage s'impose entre les jurys constitués en province et ceux de la faculté de droit de Paris et ce de part et d'autre de la zone de turbulence de la réforme du doctorat de droit en 1895.

\section{Tableau 1 : Les 23 thèses de droit public sur la représentation proportionnelle et la réforme électorale soutenues entre 1885 et 1923 (classées par ordre chronologique) ${ }^{30}$}

\begin{tabular}{|l|l|l|l|}
\hline Auteur (naissance - décès) & Titre (mention obtenue) & $\begin{array}{l}\text { Université, discipline, } \\
\text { date de soutenance }\end{array}$ & $\begin{array}{l}\text { Jury : président puis } \\
\text { membres }\end{array}$ \\
\hline 1/Campagnole Edouard & $\begin{array}{l}\text { De la représentation } \\
\text { proportionnelle dans un } \\
\text { gouvernement représentatif }\end{array}$ & Toulouse, Droit, 1885 & $\begin{array}{l}\text { Molinier, Bonfils, Paget, } \\
\text { Cabouat }\end{array}$ \\
\hline 2/Christophle & $\begin{array}{l}\text { De la représentation } \\
\text { proportionnelle }\end{array}$ & Paris, Droit, 1887 & $\begin{array}{l}\text { Jalabert, Gérardin, } \\
\text { Lefebvre, Henry }\end{array}$ \\
\hline 3/Besson Antony & $\begin{array}{l}\text { Essai sur la représentation } \\
\text { proportionnelle de la majorité } \\
\text { et des minorités }\end{array}$ & Dijon, Droit, 1897 & Deslandres ; Moulin; Roux \\
\hline 5/Faripolos Nicolas & $\begin{array}{l}\text { La démocratie et l'élection } \\
\text { proportionnelle. Étude } \\
\text { historique, juridique et } \\
\text { politique }\end{array}$ & Paris, Droit, 1899 & $\begin{array}{l}\text { Larnaude, Chavegrin, } \\
\text { Saleilles }\end{array}$ \\
\hline 6/Cameau Paul & $\begin{array}{l}\text { La représentation } \\
\text { proportionnelle dans le canton } \\
\text { de Genève }\end{array}$ & Paris, Droit, 1900 & $\begin{array}{l}\text { Chavegrin, Larnaude, } \\
\text { Leseur }\end{array}$ \\
\hline 7/Bonnefoy Gaston (1876-) & $\begin{array}{l}\text { La représentation } \\
\text { proportionnelle en Belgique } \\
\text { proportionnelle } \\
\text { (Très bien) }\end{array}$ & Caen, Droit, 1901 & E. Villey, Cabouat, Le Fur \\
\hline 8/Barriéty Clovis & $\begin{array}{l}\text { La représentation } \\
\text { proportionnelle en Belgique }\end{array}$ & Paris, Sc. écos et pols, 1902 & Sauzet, Esmein, Chavegrin \\
\hline
\end{tabular}

${ }^{30}$ Sources : outre les thèses elles-mêmes, consultées à la Bibliothèque nationale de France, des renseignements biographiques sommaires et parcellaires sur quelques étudiants parisiens ont été recueillis aux archives nationales dans les archives de la Faculté de droit de Paris (AJ 1628/AJ 1630/AJ 1645/AJ 1674/AJ 1676). Soumises à l'obligation de publication chez des éditeurs (Arthur Rousseau, Larose et Tenin, Sirey, éditeurs locaux) ces thèses sont donc conservées dans les catalogues des bibliothèques. La plupart des catalogues anciens des bibliothèques universitaires de province ayant été mis à contribution, il y a lieu de considérer cette liste de thèses comme exhaustive. Les références bibliographiques des thèses citées dans le corps du texte figurent en bibliographie finale. 


\begin{tabular}{|c|c|c|c|}
\hline 9/Dubois Paul & $\begin{array}{l}\text { La représentation } \\
\text { proportionnelle soumise } \\
\text { à l'expérience belge }\end{array}$ & Caen, Droit, 1906 & Villey, Cabant, Le Fur \\
\hline 10/Tardy René & $\begin{array}{l}\text { Les récentes propositions } \\
\text { de loi françaises tendant } \\
\text { à l'établissement de la } \\
\text { représentation proportionnelle. }\end{array}$ & Paris, Droit, 1907 & $\begin{array}{l}\text { Chavegrin, Chenon } \\
\text { Geouffre de Lapradelle }\end{array}$ \\
\hline 11/Fontaine Jean (1883-) & $\begin{array}{l}\text { La représentation } \\
\text { proportionnelle en } \\
\text { Wurtemberg (Trés bien) }\end{array}$ & Paris, Droit, 1909 & $\begin{array}{l}\text { Chavegrin, Larnaude, } \\
\text { Jacquelin }\end{array}$ \\
\hline 12/Galland Jean Justin & $\begin{array}{l}\text { La démocratie tessinoise et la } \\
\text { représentation proportionnelle }\end{array}$ & $\begin{array}{l}\text { Grenoble, Sc. pols et écos, } \\
1909\end{array}$ & $\begin{array}{l}\text { Beudant, Michoud, } \\
\text { Basdevant }\end{array}$ \\
\hline 13/Sainz François (1882-) & $\begin{array}{l}\text { Le scrutin de liste envisagé - } \\
\text { dans le système majoritaire, } \\
\text { - dans le système de la } \\
\text { représentation proportionnelle } \\
\text { (Admission) }\end{array}$ & Paris, Droit, 1909 & $\begin{array}{l}\text { Chavegrin, Larnaude, } \\
\text { Leseur }\end{array}$ \\
\hline 14/Chardon Jules Louis & $\begin{array}{l}\text { La réforme électorale } \\
\text { en France }\end{array}$ & Paris, Droit, 1910 & $\begin{array}{l}\text { Capitant, Meynial } \\
\text { Geouffre de Lapradelle }\end{array}$ \\
\hline 15/Tronqual Gaston & $\begin{array}{l}\text { La représentation } \\
\text { proportionnelle devant } \\
\text { le parlement français }\end{array}$ & Poitiers, Droit, 1910 & $\begin{array}{l}\text { Prevot-leygonie, Barrileau, } \\
\text { Bonnet }\end{array}$ \\
\hline 16/Bret Georges (1885-) & $\begin{array}{l}\text { La représentation } \\
\text { proportionnelle en Belgique } \\
\text { (Trés bien) }\end{array}$ & Paris, Sc. pols et écos, 1911 & Chavegrin, Planiol, Leseur \\
\hline 17/Jaigu Georges & $\begin{array}{l}\text { La représentation } \\
\text { proportionnelle }\end{array}$ & Rennes, Droit, 1911 & Fettu, Gidel, Rousseau \\
\hline 18/Robin Jean (1884-) & $\begin{array}{l}\text { La représentation } \\
\text { proportionnelle à l'étranger } \\
\text { dans les élections politiques } \\
\text { (récentes applications) } \\
\text { (Trés bien) }\end{array}$ & Paris, Sc. pols et écos, 1911 & Chavegrin, Leseur, Politis \\
\hline $\begin{array}{l}\text { 19/Flandin Pierre Étienne } \\
(1889-1958)\end{array}$ & $\begin{array}{l}\text { La question de la } \\
\text { représentation proportionnelle } \\
\text { en Angleterre et dans les } \\
\text { colonies anglaises. } \\
\text { Le vote transférable. }\end{array}$ & Paris, Droit, 1914 & $\begin{array}{l}\text { Geouffre de Lapradelle, } \\
\text { F. Faure, } \\
\text { J. Barthélémy }\end{array}$ \\
\hline 20/Petitjean Théodore & $\begin{array}{l}\text { La représentation } \\
\text { proportionnelle devant les } \\
\text { chambres françaises. Étude } \\
\text { d'histoire parlementaire } \\
\text { et législative }\end{array}$ & Paris, Droit, 1915 & $\begin{array}{l}\text { Larnaude, Chavegrin, } \\
\text { Barthélémy }\end{array}$ \\
\hline 21/Toupagnanine Miloche & $\begin{array}{l}\text { La représentation } \\
\text { proportionnelle }\end{array}$ & Grenoble, Droit, 1919 & Caillemer, Novel, Rouast \\
\hline $\begin{array}{l}\text { 22/Menthon François de } \\
(1900-1984)\end{array}$ & $\begin{array}{l}\text { La représentation } \\
\text { proportionnelle dans la } \\
\text { constitution fédérale Suisse }\end{array}$ & Paris, Sc. pols, 1921 & Chavegrin, Pillet, Gidel \\
\hline 23/Blavet Jean (1901- ) & $\begin{array}{l}\text { Le scrutin de liste et la } \\
\text { représentation proportionnelle } \\
\text { dans la loi du } 12 \text { juillet } 1919 \\
\text { (Bien) }\end{array}$ & Paris, Droit, 1923 & $\begin{array}{l}\text { Chavegrin ; Rolland ; } \\
\text { Mestre }\end{array}$ \\
\hline
\end{tabular}




\section{Les jurys resserrés de la faculté de droit de Paris}

À Paris, le rôle d'Ernest Chavegrin apparaît primordial autant dans la constitution des jurys que dans la pérennisation de la thématique des modes de scrutin comme pôle attractif pour les étudiants au fil des ans. Le professeur de droit constitutionnel comparé est en effet présent dans 12 des 15 jurys parisiens et se retrouve même dirigeant 9 thèses sur les 23 de notre corpus. On ne cesserait de s'interroger sur l'intérêt de Chavegrin pour la représentation proportionnelle si l'on ne savait par ailleurs la fonction centrale qu'il a pu occuper dans le système doctoral parisien de l'époque en encadrant un nombre incroyable de thèses ${ }^{31}$. L'intérêt de Chavegrin pour les aspects parlementaires de la réforme du mode de scrutin est constant. Il fait soutenir deux thèses prétendant au commentaire des débats législatifs français : l'une en 1907 ( $\mathrm{n}^{\circ} 10, \mathrm{R}$. Tardy) ${ }^{32}$ au moment où s'inaugure ce débat, l'autre en 1923 pour faire le bilan de la loi de 1919 instaurant en partie la représentation proportionnelle ( $n^{\circ} 23$, J. Blavet). Entre ces deux dates, il dirige essentiellement des travaux consacrés aux exemples étrangers (belge, suisse) d'application de la représentation proportionnelle (thèses $\mathrm{n}^{\circ} 5,8,11,16$ et 18) faisant ainsi correspondre plus explicitement ces recherches avec son programme d'enseignement de droit constitutionnel comparé. L'importance d'E. Chavegrin est particulièrement visible dans le binôme académique qu'il forme avec Ferdinand Larnaude. Ce dernier ne participe à aucun jury dans lequel le premier ne figure pas. L'invitant deux fois (notamment pour la thèse déjà citée de N. Saripolos) il se voit rendre la pareille quatre fois. Cette relation permet ainsi à Larnaude, militant proportionnaliste et aspirant éternel à une carrière électorale, de rendre visible l'expertise rationalisée et contradictoire que la science juridique est alors en mesure de construire ${ }^{33}$. C'est sans doute également pour cela qu'il invite Adhémar Esmein, farouche opposant à la représentation proportionnelle ${ }^{34}$, au jury de thèse de N. Saripolos.

Plus généralement, deux configurations collectives structurent les jurys. Premièrement, la réforme électorale comme enjeu conjoncturel peut autoriser des jurys plus « politiques » que d'autres. On ne peut par exemple pas comprendre autrement la thèse que dirige Marc Sauzet en 1902 entre deux mandats de député de l'Ardèche (thèse $\mathrm{n}^{\circ} 7 \mathrm{de} \mathrm{G}$. Bonnefoy nettement hostile à la pratique de la proportionnelle). Revenu à l'université après avoir démissionné de la députation en 1899 mais aucunement spécialiste des questions électorales (ni, plus généralement, de droit constitutionnel), il s'entoure à la fois d'Adhémar Esmein, également hostile, et d'E. Chavegrin, qui n'en est alors qu'à son deuxième jury en la matière. L'autre exemple frappant de cette concordance des dispositions politiques et universitaires nous est donné par le cas de Louis Rolland. Agrégé depuis 1906, ce n'est pourtant qu'en 1923 qu'il participe à un jury... soit quelques années seulement avant son entrée à la Chambre des députés en

31 SACRISTE, 2002, tome 1, 269 -270.

${ }^{32}$ Le numéro indique la position chronologique de la thèse (de 1 à 23) et se réfère au tableau de présentation du corpus.

${ }^{33}$ Larnaude est à l'intersection de l'expertise (il fonde la Revue du droit public et de la science politique en 1894) et de la politique : maire de Condom dans le Gers, il se présente vainement en 1898, 1902 et 1905 aux élections législatives. Cf. Bigot, 2007. Sur l'influence des défaites récurrentes de certaines fractions des élites de la République dans leur conversion au « commentaire électoral », cf. MARTY et SCHWARTZ, 2008.

${ }^{34}$ Esmein, 2001, 318. 
1928 comme représentant du Maine-et-loire ${ }^{35}$.

Secondairement, la participation à un jury sur la proportionnelle peut souvent s'inscrire, a posteriori, comme un point de passage pour ceux qui seront appelés à occuper dans le futur des chaires stratégiques de droit constitutionnel pour lesquelles cette question, et les problèmes pratiques qu'elle met en perspective (théories de la souveraineté, évolutions des démocraties représentatives d'Europe, transformations des «partis » politiques), pourrait servir de point d'appui non négligeable. Trois personnalités du droit constitutionnel de l'entre-deux-guerres entrent dans cette catégorie. En tout premier lieu, Joseph Barthélémy participe à deux jurys au tout début de sa carrière parisienne en 1914 et 1915. Invité par Larnaude ou Geouffre de Lapradelle, il fait valoir ses compétences directes d'expert sur la représentation proportionnelle. Albert Geouffre de Lapradelle qui assure le cours de droit constitutionnel de Licence de 1907 à 1939 n'est pas moins sensible à la tendance de ces thèses à publiciser le problème de la représentation proportionnelle. Assistant à la thèse d'histoire parlementaire immédiate de R. Tardy en 1907 il ira même jusqu'à diriger celle de Pierre Étienne Flandin en 1914. On saisira d'autant plus l'opportunité de ces rencontres académiques qu'à la même époque son cours comporte lui-même une partie très volontariste sur les modes de scrutin dont le sens profond est explicite : « (...) autant le mécanisme de la représentation proportionnelle peut sembler long et difficile à décrire, autant il est simple à appliquer. Rien n'est plus complexe que le mécanisme d'une montre : quoi de plus facile à remonter ? $»^{36}$. Assistant à deux jurys de thèse assez militants en 1911 ( $\mathrm{n}^{\circ}$ 17, Georges Jaigu) et 1921 ( $\mathrm{n}^{\circ}$ 22, François de Menthon), Gilbert Gidel développe un point de vue quasiment identique. Bien que plutôt défavorable au principe de la représentation proportionnelle (suivant Esmein, il indique que ce mode de scrutin est contraire à la théorie du gouvernement représentatif où " le député a pour fonction d'exprimer la volonté de la nation prise dans son unité indivisible $\|^{37}$ ), il évacue cependant ce problème théorique pour se prononcer positivement sur l'opportunité politique de défendre ce système.

\section{Des jurys d'entrepreneurs constitutionnels en province}

On peut retrouver dans le corpus des présidents de jury de province la plupart des noms de ceux qui seront les acteurs de la révolution conservatrice dans le champ doctrinal du droit constitutionnel, au nom d'un « droit naturel» spiritualiste et chrétien, à la veille de la première guerre (Edmond Villey, Robert Beudant...) ${ }^{38}$. Ce mouvement entendait renouveler la doctrine et l'enseignement du droit constitutionnel par une critique de l'État républicain et de ses omnipotences comme par exemple le parlementarisme. Dans cette nouvelle fonction revêtue par la doctrine l'appui des thèses joue un rôle non négligeable. En effet, la question de la représentation proportionnelle comme

\footnotetext{
${ }^{35}$ Sur l'engagement électoral des juristes à cette époque, cf. MILET, 2000.

${ }^{36}$ Geouffre de lapradelle, 1912, 202.

${ }^{37}$ GidEL, 1938 - 1939, 160.

${ }^{38}$ Nous suivons en cela les hypothèses de SACRISTE, 2002, tome 2, chapitre 6. Pour connaître le contenu des cours non publiés ou à diffusion discrète, nous avons eu recours aux documents conservés par les archives nationales, F/17/13141-13156. Pour la faculté de droit de Paris et/ou la période ultérieure, la diffusion imprimée des cours et manuels se suffit à elle-même.
} 
enjeu saillant de la vie politique encourage les professeurs de droit constitutionnel à intervenir hors de l'univers académique afin de valider cette image " d'ingénieur $d u$ social sur la base de ressources scientifiques $\|^{39}$. Deux figures du droit constitutionnel provincial de la fin du XIX ${ }^{\mathrm{e}}$ siècle peuvent tout d'abord nous intéresser pour des raisons sensiblement équivalentes : Victor Molinier à Toulouse et Maurice Deslandres à Dijon qui semblent tous les deux précurseurs en matière de direction de thèses " généralistes » sur la représentation proportionnelle. En 1885, Molinier dirige le travail d'Édouard Campagnole dont la thèse de droit français (« De la représentation proportionnelle dans un gouvernement représentatif ») est sans doute le premier travail de mise au point systématique des aspects historiques, théoriques et techniques du mode de scrutin proportionnel. Son travail est d'autant plus novateur que sa thèse (sans la partie de Droit romain) est publiée sous un titre légèrement différent chez l'éditeur parisien Pichon $^{40}$ qui publiera trois années plus tard le volume de la Société pour l'étude de la représentation proportionnelle. Sans être un entrepreneur constitutionnel de premier ordre, on sait qu'en 1885 au moins le cours de V. Molinier comportait une partie entière consacrée à l'électorat conçu comme le premier des quatre acteurs de la séparation des pouvoirs. Les théories liées à la représentation des minorités (système d'Hare, apparu 20 ans avant) y étaient fortement développées ${ }^{41}$.

Le titre d'entrepreneur constitutionnel ne peut par contre pas se discuter pour Maurice Deslandres. Son entreprise de redéfinition du droit public repose en effet autant sur des exigences générales de méthode (à travers la polémique sur la crise de la science politique ${ }^{42}$ ) que sur des implications constitutionnelles et politiques dont la revendication " proportionnaliste » n'est pas la moins mise en avant. La thèse qu'il dirige en 1897 ( $\mathrm{n}^{\circ} 3$, Antony Besson) lui permet d'ailleurs de concilier ces deux objectifs. Elle est d'abord plus complète que celle d'E. Campagnole au niveau de la description des différentes modalités de représentation proportionnelle. Là où l'étudiant toulousain se contentait de considérer les mécanismes de formation de la majorité et de la minorité sous l'emprise de la proportionnelle, A. Besson approfondit lui de manière séparée et pointue les différents systèmes de formation des listes, la nature du vote émis selon le type de liste et enfin les divers procédés mathématiques de comptabilisation des voix et des sièges. Il articule ensuite son discours à la nécessité politique pour la Troisième République d'adopter la représentation proportionnelle « ici et maintenant ». Surtout, la thèse d'A. Besson apparaît à l'instar de celle de N. Saripolos en 1900 comme un bien doctrinal pouvant circuler entre les professeurs eux-mêmes afin de réévaluer leurs positions respectives en matière d'expertise constitutionnelle. C'est dans cette optique que la thèse dirigée par $\mathrm{M}$. Deslandres permettra explicitement à son ancien

\footnotetext{
${ }^{39}$ Ibid, 657.

${ }^{40}$ Campagnole, 1885 . Le même éditeur publie cette année-là le cours élémentaire de droit constitutionnel de V. Molinier.

${ }^{41}$ Le programme des cours figure dans Académie de Toulouse, 1885. Sur la faculté de droit de Toulouse à la fin du $19^{\mathrm{e}}$ siècle et sur la conception du droit naturel chez Molinier, cf. BuRneY, 1988, 133-146. Molinier est professeur de législation criminelle et pénale de 1843 à 1881. Il a laissé toutefois un Cours élémentaire de droit constitutionnel indiqué comme inachevé (cf. HALPÉRIN, 2007 b, 568 - 569).

${ }^{42}$ La genèse et les conséquences des articles de Deslandres dans la Revue du Droit public sur la « crise de la science politique en France » sont étudiées par SACRISTE, 2001, 69-94.
} 
collègue de Dijon et ami R. Saleilles de produire le premier article conséquent de la doctrine constitutionnelle à partir d'un long compte-rendu du travail d'A. Besson ${ }^{43}$ qui correspond donc en tout point à la perspective historiciste défendue par ses deux maîtres dijonnais.

Cette figure de l'expertise constitutionnelle apparait encore plus clairement à la Faculté de droit de Caen où enseignent deux autres professeurs à mi-chemin, comme M. Deslandres, du conservatisme et du catholicisme social : Edmond Villey et Louis Le Fur. La critique politique évoquée en filigrane chez Deslandres prend chez eux la forme d'une entreprise de longue haleine qui essaie de fonder en théorie et en pratique la nécessité du passage à la proportionnelle. La première forme de ce militantisme chez E. Villey est directement en prise avec les deux thèses qu'il dirige en 1901 et 1906 sur la représentation proportionnelle en Belgique ( ${ }^{\circ} 6$, P. Cameau; $n^{\circ} 9$, Paul Dubois). Il publie en effet en 1900 un volume de synthèse sur les différents systèmes électoraux d'Europe qui comporte, entre autres, un important chapitre sur la « représentation proportionnelle des minorités et des intérêts ». Malgré une prise de position limitative d'entrée ( «...il n'est assurément pas désirable que les opinions les plus malsaines soient représentées dans les Chambres » - il pense ici aux socialistes -), ce texte est une véritable matrice des critiques de la démocratie représentative que la revendication de la proportionnelle permet de faire émerger : abstention grandissante, déclin de l'autorité parlementaire, grandes décisions en fait minoritaires, instabilité et abus de pouvoir des gouvernements ${ }^{44}$. Ce réquisitoire inaugure également, par la synchronisation des différentes crises de la démocratie en Europe qu'il présuppose, l'essai d'importation symbolique de la représentation proportionnelle en France. Les deux thèses que Villey dirigera auront alors pour but de " prendre exemple » sur la Belgique et sur la dynamique de son réformisme du mode de scrutin influencé par le parti catholique. Pour contrer le mode de scrutin majoritaire, Villey s'attaque à ce qu'il estime en être le fond doctrinal : la théorie de la souveraineté nationale telle qu'elle se développe en France de Rousseau jusqu'à A. Esmein. Le livre qu'il publie en 1910, "Les périls de la démocratie », n'expose pas clairement la revendication de la proportionnelle mais en fournit une base théorique solide : c'est parce que l'on accorde avec excès un droit de commandement à l'État hors de tout respect de la liberté individuelle qu'il faut dès lors considérer cette dernière comme la base de la représentation. Villey critique explicitement le fait que les grandes décisions du bloc des gauches (il cite la loi sur les associations de 1901, la loi de séparation de 1905, les retraites ouvrières) aient été en fait des décisions minoritaires qui, s'incarnant trompeusement dans une majorité, empiètent sur la liberté sociale de l'individu ${ }^{45}$. E. Villey réitère cette critique quelques années plus tard à la fin de la première guerre mondiale en 1918. Renouvelant ses critiques au moment du remplacement de la chambre élue avant la guerre en 1914, sa volonté n'est plus simplement de théoriser une conception alternative de la souveraineté mais de proposer une réforme constitutionnelle dans laquelle il importe de donner corps à la réglementation du suffrage universe ${ }^{46}$. Mais en 1918 la fracture

\footnotetext{
${ }^{43}$ SAleilles, 1898.

${ }^{44}$ Villey, 1900, 127-128.

${ }^{45}$ Villey, 1910.

${ }^{46}$ Villey, 1918, 9.
} 
entre ce que propose E. Villey et la nature des produits politiques concourants est patente. Alors que la représentation proportionnelle est partout défendue au nom de l'exigence d'une plus grande justice collective dans la répartition des opinions, le professeur de Caen s'oppose nettement à cette idée : la représentation proportionnelle qu'il désire tout aussi ardemment doit être le moyen de retrouver la plénitude de la souveraineté individuelle et de la liberté sociale et politique qu'elle requiert. Si l'accord politique se fait donc entre tous les proportionnalistes pour dénoncer les dérives " favoritistes » du scrutin majoritaire, E. Villey est isolé dans sa tentative de fonder la proportionnelle sur une théorie du gouvernement représentatif qui limiterait le pouvoir des représentants et des représentés ${ }^{47}$.

\section{Travail d'écriture et politisation des thèses sur la réforme électorale}

La médiation des jurys de thèse permet donc de comprendre la double dépendance qui anime le travail juridique dans sa participation au débat sur la pertinence de l'adoption de tel ou tel mode de scrutin. Ne possédant pas de doctrine constituée (à part quelques entreprises éparses agissant à différents niveaux plus ou moins académiques : E. Villey, J. Barthélémy, R. Saleilles...), les professeurs de droit (constitutionnel ou non) s'en remettent aux thèses faites sous leur direction pour constituer une expertise conséquente. Cette première dépendance en appelle une seconde : ces thèses, sommées d'être collectivement visibles, se limitent le plus souvent à reproduire les mises en forme militantes ou parlementaires, extra-académiques en tout cas, qui contribuent à dessiner un processus de réforme électorale. Elles ne le font certes pas de la même manière selon que l'on se situe dans les années 1880 ou 1920, d'abord et essentiellement parce que l'encadrement professoral et intellectuel du doctorat en droit s'est profondément transformé mais aussi en fonction de l'avancement politique des projets de réforme électorale. La distribution temporelle de ces travaux est elle-même sensible à cette conjoncture et invite à distinguer différentes formes de thèse. Vers 1900, l'ambition est de produire des thèses généralistes visant à justifier l'idée même de représentation proportionnelle par rapport au principe majoritaire dans ses dimensions historiques, juridiques voir philosophiques. À partir de 1900 et jusque vers 1914 la tendance est à la description d'expériences étrangères qui sont alors conçues, directement ou indirectement, comme des méthodes à suivre pour accomplir la réforme électorale en France. Enfin, à partir de 1907 et jusqu'en 1923, les travaux s'orientent vers l'étude des différentes étapes parlementaires du processus de réforme électorale tel qu'il se produit en France ${ }^{48}$.

\footnotetext{
${ }^{47}$ Sur ce point, cf. VILLEy, 1918, 46-54. Le Fur approfondira quelques années plus tard cette vision d'une crise généralisée du lien représenté/représentant dans lequel, paradoxalement, la question initiale du mode de scrutin passe au second plan (LE Fur, 1934, 7-49). La conception élitiste et anti-majoritaire de Le Fur est rappelée par Milet, 2007, 485-486.

${ }^{48}$ Le choix de cette période 1885-1923 est strictement dépendant du niveau quantitatif de production doctrinale. Il semble en effet ne pas y avoir de thèses sur ces sujets avant 1885 et elles deviennent quasiinexistantes à notre connaissance après 1923.
} 


\section{Les droits d'entrée dans un champ d'étude spécifique}

La recherche des fondements de la représentation proportionnelle concerne 5 thèses soutenues entre 1885 et $1902\left(\mathrm{n}^{\circ} 1,2,3,4\right.$ et 7$)$ et 2 autres un peu plus tardives $\left(\mathrm{n}^{\circ} 17,21\right)$ mais deux travaux (A. Besson et N. Saripolos) se détachent par l'ampleur de leur documentation et la réception qu'ils suscitent. La thèse de N. Saripolos (1899) semble particulièrement centrale tant par son volume inégalé (1000 pages) que par l'importance stratégique de son jury (F. Larnaude et R. Saleilles, militants de la cause proportionnaliste; E. Chavegrin, cheville ouvrière des jurys de thèse sur la représentation proportionnelle). Elle contribue surtout à forger l'identité et la spécificité du travail des juristes parmi l'éventail des expertises en matière de modes de scrutin. C'est ainsi que la recherche des origines juridiques de la représentation proportionnelle est supposée apporter des justifications « supérieures » à la revendication de son opportunité politique. C'est pour cela qu'il entend clarifier sa position initiale par tout un arsenal de concepts relativement nouveaux (empruntés à Aristote notamment). Il distingue tout d'abord trois formes de gouvernement : la démocratie directe, la démocratie semi-représentative et la démocratie représentative pure qu'il s'empresse de renommer " démocratie complexe ». Dans cette dernière, le citoyen n'a qu'un "droit réflexe venant de l'État ${ }^{49}$ ne lui octroyant qu'un droit de nomination, de désignation de ses représentants et de ses gouvernants. Autrement dit le citoyen ne peut être tenu pour un législateur en puissance et reste un simple électeur : l' « organe élisant $\aleph^{50}$ ne saurait être considéré comme un tout et encore moins comme un tout décidant au nom de tous. Or, toujours selon Saripolos, le scrutin majoritaire comme la représentation proportionnelle (qu'il assimile à une représentation personnelle dans laquelle la souveraineté serait divisible mais non fongible) aboutissent à des formes semi-représentatives de gouvernement dans lesquelles l'électeur est abusivement assimilé à un " législateur ». Il écarte donc d'emblée certains fondements théoriques importants défendus par ceux qui réclament la représentation proportionnelle au nom d'une représentation personnelle conçue comme "carte réduite de la nation " ${ }^{51}$. Il affirme d'ailleurs que la proportionnalité parfaite n'est qu'un idéal impossible à atteindre et que l'on doit se borner à réclamer et améliorer la "participation de tous aux affaires publiques $»^{52}$. Peu importe finalement la construction juridique préalable qui vise à remplacer la « représentation proportionnelle » par « l'élection proportionnelle » (concept qui correspond mieux à sa vision des droits de l'électeur) : ce n'est pas tant la proportionnalité qui intéresse Saripolos mais la refonte du rapport de représentation entre l'électeur et l'élu par la clarification des rôles respectifs de chacun : l'électeur doit être plus libre et ne pas être obligé de choisir entre des coalitions douteuses, le député doit être plus indépendant et généré une confiance clairement basée sur ses idées.

À partir de prémices théoriques opposés à ceux de Saripolos, la thèse d'A. Besson aboutit à peu près à la même volonté de maîtrise des luttes électorales. Dans la thèse de l'élève de M. Deslandres, chaque électeur se définit par son unicité et l'ensemble des

\footnotetext{
49 SARIPOLOS, 1899, 595.

${ }^{50}$ Ibid, 610.

${ }^{51} \mathrm{Ibid}, 513$. L'expression fait référence à un discours de Mirabeau.

${ }^{52}$ Ibid, 624.
} 
électeurs par leur égalité : les citoyens étant réellement porteurs d'une part identique de souveraineté individuelle il est nécessaire que "les différentes opinions aient dans les corps élus une importance proportionnelle à celle qu'elles ont dans le pays ${ }^{53}$. La souveraineté nationale n'est donc pas comme chez Saripolos ce bien collectif indivisible qui interdit à chaque individu de s'en prévaloir. Et c'est justement parce que «le droit de chaque citoyen est absolu $\|^{54}$ que la proportionnalité de la représentation est pour l'auteur une préoccupation centrale. Dans le cadre d'un collège électoral uninominal (cas assez rare) le système majoritaire est parfaitement proportionnel : la moitié plus un suffit à assurer le principe d'égalité des électeurs. Mais l'application de ce principe à un collège pluri-nominal (c'est-à-dire selon Besson à toute élection législative dans le système français d'alors) suppose l'abandon du système majoritaire et l'introduction de la représentation proportionnelle proprement dite. La représentation proportionnelle est alors revendiquée au nom d'un nécessaire perfectionnement des outils permettant de clairement délimiter une " majorité » d'une " minorité ». Au niveau de l'électeur et donc de la répartition des votes, A. Besson étudie avec précision la question des inégalités des circonscriptions et donc des seuils de voix nécessaires pour être élu ${ }^{55}$. $\mathrm{Au}$ niveau de l'élu et donc de la répartition des sièges à pourvoir, il développe une « théorie de la concurrence des listes » qui occupe 100 des 300 pages de son ouvrage. Ces deux thèses sont donc les deux principales recherches sur les origines juridiques de la représentation proportionnelle mais leur postérité renvoie également aux formes de revendication politique qu'elles défendent. Cette dualité encadre bien le processus d'autonomisation de cette question de la représentation proportionnelle dans l'ensemble de la doctrine constitutionnelle. Les coûts d'entrée dans ce secteur disciplinaire sont doubles : si une « compétence » théorique particulière est nécessaire pour délimiter le champ spécifique de la représentation proportionnelle par rapport au scrutin majoritaire, une « appétence » s'avère tout aussi indispensable pour prospecter les solutions pratiques et les conséquences inhérentes à l'introduction de cette valeur cardinale qu'est la proportionnalité de la représentation ${ }^{56}$.

\section{L'« importation » des exemples étrangers de représentation proportionnelle}

Relevant a priori de l'observation des évolutions réelles du droit public, les thèses relatant des expériences étrangères s'avèrent surtout des réflexions substitutives au débat français (qui n'aboutit à aucune loi avant 1919). Les deux thèses sur la représentation proportionnelle en Belgique dirigées par E. Villey à Caen le prouvent parfaitement. Les deux travaux se félicitent également de l'adoption de la représentation proportionnelle par la Belgique en 1899 et prétendent l'ériger en exemple pour la France. Mais la première thèse, celle de $\mathrm{P}$. Cameau $\left(\mathrm{n}^{\circ} 6\right)$, se contente de présenter le cheminement historique suivi et donc à suivre : "l'histoire de la représentation proportionnelle en Belgique, (...) peut présenter un certain intérêt, car il est possible d'en tirer quelques

\footnotetext{
${ }^{53}$ Besson, 1897, 39.

${ }^{54} \mathrm{Ibid}, 30$.

${ }^{55}$ Ibid, $75-88$.

${ }^{56}$ Sur les notions de « compétence » et d' « appétence » dans l'établissement des coûts d'entrée dans une discipline scientifique, cf. Bourdieu, 2001, 101.
} 
indications sur la manière dont doit être organisée la propagande $\|^{57}$. La deuxième thèse, celle de P. Dubois ( $\mathrm{n}^{\circ}$ 9), participe quant à elle, quelques années plus tard, explicitement du contexte de mobilisation en faveur de la représentation proportionnelle : " cette étude est en définitive le fruit d'une enquête personnelle faite sur les lieux qui nous a permis (...) de constater les avantages réels qui ont résulté de l'application de ce système électoral $»^{58}$. On peut même constater que le plan de cette thèse est calqué sur celui d'une brochure d'Eugène Duthoit ${ }^{59}$, professeur de droit constitutionnel à la faculté libre de droit de Lille, qui rédigera d'ailleurs un compte rendu du travail de Dubois dans son journal militant « Le proportionnaliste $»^{60}$. C'est donc assez directement que P. Dubois préconise de suivre l'exemple belge et même d'emprunter leur principe de proportionnalité, dit système d'Hondt. Etudiant de Villey et proche de Duthoit, deux personnalités du droit constitutionnel dont les convictions catholiques sont fortes, P. Dubois est sans doute également sensible à la manière dont la réforme belge a assuré la pérennité du parti catholique (alors que ce même parti était opposé à la représentation proportionnelle dans les années 1890).

La sensibilité à des expériences étrangères de représentation proportionnelle ne se limite pas bien sûr au cas belge de 1899. Cette phase de croyance s'alimente de toutes les expériences favorables à la représentation proportionnelle qui peuvent éclairer le champ politique français sur la marche à suivre. Les thèses soutenues sous la direction d'E. Chavegrin sont particulièrement intéressantes de ce point de vue. Elles dessinent les différentes formes de cette importation symbolique de la réforme électorale. Elle peut d'abord emprunter des voies détournées soit en utilisant la monographie pour établir une théorie générale et évolutionniste de l'opposition entre principe majoritaire et principe proportionnel (c'est le cas en 1900 de la thèse de A. Fournier à partir de l'application de la représentation proportionnelle à Genève en 1892), soit en rassemblant des expériences disparates de plusieurs pays dans une vision synchronisée présentant l'introduction de la représentation proportionnelle comme une conséquence inévitable quelques soient les conditions locales initiales (thèse $\mathrm{n}^{\circ} 18$ ). L'importation peut aussi être plus explicite en se servant du cas étranger uniquement comme prétexte. C'est le cas de la thèse de $J$. Fontaine $\left(\mathrm{n}^{\circ} 12,1909\right)$ sur la représentation proportionnelle dans le Land allemand du Wurtemberg qui ne se consacre à son sujet que dans la deuxième partie du texte et réserve la première à un " parallèle entre le système majoritaire et la représentation proportionnelle ». Il peut ainsi annoncer d'emblée son objectif : «... si la représentation proportionnelle apparaît comme le système qui répond le mieux dans tel pays aux exigences du gouvernement parlementaire (...) il convient alors de rechercher si son introduction dans tel autre pays se heurte à des obstacles insurmontables (...) malgré les différences qui résultent de la tradition, du tempérament et des habitudes de race ${ }^{61}$. C'est également le cas de J.-J. Galland $\left(\mathrm{n}^{\circ} 14\right)$ qui tire de sa thèse sur la représentation proportionnelle dans le canton suisse du Tessin une brochure politique revendiquant la représentation proportionnelle pour la France : arguant de l'œuvre de

\footnotetext{
${ }^{57}$ Cameau, 1901, 98.

${ }^{58}$ Dubois, 1906, 13.

${ }^{59}$ Duthoit, 1904. (L'éditeur est le même que celui de Dubois).

${ }^{60}$ Le proportionnaliste, $1906,87$.

${ }^{61}$ Fontaine, 1909, 6.
} 
pacification produite en Suisse il prétend user de ce savoir juridique pour influencer le cours du débat parlementaire en France : "La question est donc enterrée à la Chambre; mais elle reviendra dans quelque mois devant le pays, qui lui donnera certainement une solution conforme aux vues de ceux qui veulent introduire dans nos lois plus de raison et plus de justice $»^{62}$. Tout autant que les réalités politiques des pays étudiés, ces thèses s'avèrent dépendantes de la richesse des travaux intellectuels qui y sont produits à propos des modes de scrutin dès les années 1870 - 1880 et dont elles tentent une forme d'importation symbolique 10 ou 20 ans après dans un débat intellectuel français tout entier dominé par des publicistes aspirants plus à une carrière électorale qu'académique. Si l'université française élabore au tournant des années 1900 une stratégie d'exportation dans toute une série de disciplines (lettres, histoire, archéologie $)^{63}$, le droit public - y compris sous la forme du droit comparé - apparaît plutôt ici comme un pôle d'importation.

\section{La codification intellectuelle de l'ordre parlementaire de la réforme électorale}

De cette dynamique d'importation on retiendra principalement la focalisation sur la chronologie parlementaire de la controverse. On passe donc d'études à prétention historique sur la « représentation proportionnelle » à des études plus politiques sur la notion de « réforme électorale». Cette transformation peut être suivie à travers quelques thèses qui permettent de reconstruire la genèse parlementaire de ce débat des premières propositions à la fin de la législature 1902-1906 jusqu'à l'adoption de la loi du 12 juillet 1919. La première thèse consacrée à la réforme électorale française n'intervient qu'en 1907 : elle est l'œuvre de R. Tardy ( $\left.{ }^{\circ} 10\right)$ au moment de la première véritable mobilisation parlementaire pour la représentation proportionnelle. Pour autant, ce travail n'est pas réellement ancré dans l'étude des débats parlementaires mais a plutôt pour objectif d'accompagner les premiers pas de la représentation proportionnelle dans cette arène. Ses sources sont doubles : d'une part la mise en exergue des livres et brochures produits par les militants extra-parlementaires de la cause, d'autre part l'étude des prises de position des différents « partis » à travers les actes de leurs congrès. Par l'exemplarité de l'emploi de sources non strictement juridiques, le militantisme de cette thèse apparaît moins clairement que dans celles qui lui succèderont. Il se résume simplement à anticiper ce que pourrait être le comportement des différentes tendances politiques face à des systèmes électoraux qui arbitreraient, dans un sens ou l'autre, le poids respectif du candidat ou du «parti » appelés à être les acteurs de la formation des listes. Ne se prononçant pas explicitement en faveur de la représentation proportionnelle, le travail de $\mathrm{R}$. Tardy contribue toutefois à accroître la visibilité de la mise en forme parlementaire de cette cause. Ces thèses vont donc plus largement contribuer à imposer le calendrier parlementaire de la réforme électorale. La thèse soutenue à Poitiers en 1910 par G. Tronqual ( $\left.n^{\circ} 15\right)$ adopte un plan classique tout à fait semblable à ceux de N. Saripolos ou A. Besson (recherche d'un fondement juridique à la représentation proportionnelle tout d'abord, classification des différents systèmes ensuite, analyse coûts/avantages enfin) mais le fait reposer sur une articulation historique

\footnotetext{
${ }^{62}$ Galland, s. d 44.

${ }^{63}$ Charle, 2004, 323-347.
} 
qui relate strictement, c'est-à-dire législature par législature, les avancées et reculées de la représentation proportionnelle devant la Chambre des députés. Cette généralisation d'une histoire uniquement parlementaire de la revendication de la représentation proportionnelle est poussée à son paroxysme dans la thèse de J.-L. Chardon qui prend d'ailleurs pour titre « la réforme électorale en France » $\left(\mathrm{n}^{\circ} 14\right)$. Produite au plus fort de la controverse parlementaire en 1910 (un vote parlementaire de novembre 1909 et les élections législatives d'avril 1910 laissaient à penser que la représentation proportionnelle pourrait être assez rapidement adoptée), la thèse en restitue les différentes phases de mobilisation. Il n'est plus question d'anticiper le comportement des députés vis-à-vis de la réforme mais de jeter un double regard rétrospectif sur le travail accompli par le parlement : d'une part très immédiatement en compilant les votes émis lors de la discussion de tel ou tel article de la proposition contenue dans le rapport du député Étienne Flandin déposé en $1907^{64}$, d'autre part en faisant remonter les origines du traitement parlementaire de la question de la représentation proportionnelle jusqu'à la Révolution Française (historique inédit hormis chez N. Saripolos). Au final la contribution nette de Chardon à l'avancement de la cause consiste à autonomiser le rôle de l'éducation parlementaire : "pour mesurer les chances de la réforme, ce n'est pas l'esprit du corps électoral, c'est celui du parlement qu'il faut considérer ${ }^{65}$.

En poussant un peu plus loin le curseur historique, la thèse de T. Petitjean $\left(n^{\circ} 20\right)$ conserve la même croyance dans la centralité parlementaire mais en lui associant une prise de position très défavorable à la représentation proportionnelle (alors même que le président du jury est F. Larnaude). Cette thèse dont le sous-titre est explicite, " essai d'histoire parlementaire et législative ", tend à prouver que la compétence scientifique nécessaire à toute étude juridique sur la représentation proportionnelle n'est plus l'alignement dans le champ des produits professoraux (Petitjean ne fait que de rares allusions à A. Esmein alors même qu'il reprend plus ou moins sa justification du scrutin d'arrondissement) mais la restitution des conditions du jeu parlementaire. Si la thèse précédemment analysée de J.-L. Chardon laissait à penser que l'issue inévitable du débat sur la représentation proportionnelle était la « réforme électorale », celle de T. Petitjean considère au contraire que certaines de ces évolutions invalident tout processus de changement des règles du jeu. En clair, les successions de propositions, de rapports et les dizaines de séances plénières ne sont pas vues comme le point de départ d'une dynamique de réforme mais comme la conséquence de son impossibilité structurelle : «... la Chambre jusqu'à ce jour, cependant en bonne majorité proportionnaliste, s'est montrée impuissante à arrêter un texte $»^{66}$. Au fur et à mesure de l'accumulation de ces thèses leur dépendance à l'égard de la conjoncture parlementaire s'accroît. Ainsi en 1914, T. Petitjean consacre 30 pages aux aléas de la représentation proportionnelle entre 1873 et 1906 et pas moins de 150 aux deux dernières législatures (1906-1910 et 1910-1914). La problématique du mode de scrutin devient presque une commodité pour comprendre une histoire politique immédiate plus générale. La thèse de $\mathrm{J}$. Blavet qui vient clore chronologiquement cet ensemble d'études évoque ainsi non seulement la genèse parlementaire de la loi du 12

\footnotetext{
${ }^{64} \mathrm{Cf}$. le tableau de compilation des votes par tendance politique (CHARDON, 1910, 247).

${ }^{65}$ Ibid, 252.

${ }^{66}$ Petitjean, 1915, 247.
} 
juillet $1919^{67}$ mais aussi son application aux élections d'après-guerre de novembre 1919. À lire ce travail, on observe que le contexte de guerre et plus encore de rétablissement de la paix oblige l'auteur, mais il n'est évidemment pas le seul à l'époque, à considérer la représentation proportionnelle comme la "préface d'une réforme plus vaste ${ }^{68}$.

\section{Conclusion. Une contribution au marché du commentaire électoral}

On ne pourrait pas terminer ce panorama des thèses sur la représentation proportionnelle sans noter la présence dans notre corpus de deux étudiants un peu particulier: Pierre Étienne Flandin et François de Menthon. Outre les points communs qu'ils possèdent de par deux thèses consacrées à de courtes études sur des expériences étrangères de représentation proportionnelle (l'Angleterre et la Suisse), c'est leurs parcours politiques ultérieurs (dans l'entre-deux-guerres, pendant la guerre et même au début de la IVe République) qui peut sans doute les réunir et révéler certains des usages de l'expertise acquise en matière de mode de scrutin. Ainsi en 1919, le futur président du conseil P. E. Flandin est élu député de l'Yonne par le scrutin même qu'il a défendu dans sa thèse cinq ans auparavant. De même, 25 ans après sa thèse, le ministre de l'économie du gouvernement Bidault, F. de Menthon, continue à revendiquer à l'aube de la $I V^{e}$ République la représentation proportionnelle comme moyen de rationaliser le système partisan ${ }^{69}$. De la continuité des prises de position on ne déduira pas, bien entendu, une hypothétique intensité de l'investissement intellectuel à propos des modes de scrutin ni même, plus spécifiquement, de l'investissement dans le travail d'écriture de la thèse. Pour F. de Menthon en effet, on sait « que les années passées sur les bancs de la faculté de droit ne lui [ont] pas laissé de souvenir prégnant $\gg^{70}$. Cette période est surtout marquée pour lui par l'apprentissage du militantisme au sein de l'Association catholique de la jeunesse française ${ }^{71}$. Ses premiers pas en politique sont donc toutefois accompagnés d'une réflexion précoce et impersonnelle sur les moyens de construire et de pérenniser une carrière électorale.

On voit donc à quel point la production de thèses de droit sur la « représentation proportionnelle » et la " réforme électorale » oscille sans cesse entre la nécessaire exploration d'un produit constitutionnel permettant de s'inscrire dans le débat doctrinal et la volonté de mettre en forme de nouvelles justifications de la délégation électorale. Inauguré par une volonté de refonder juridiquement (donc théoriquement) le rapport de représentation entre l'électeur et l'élu, le mouvement que dessine l'ensemble de ces thèses est, en fin de compte, celui d'un renforcement des éléments constitutifs (l'électeur, le parti et le parlement) de l'« illusion bien fondée $»^{72}$ qu'est la démocratie représentative réduite à sa dimension pratique de délégation électorale.

${ }^{67} \mathrm{Il}$ s'agit en fait d'un scrutin de liste départemental à 1 tour où la proportionnelle ne joue que pour les candidats non élus à la majorité absolue.

${ }^{68}$ BLAVET, 1923, 3.

${ }^{69}$ De menthon, 1946, 35.

${ }^{70}$ Ibid.

${ }^{71}$ Lacroix, 1985, 175-185.

${ }^{72}$ Lacroix, 1985, 175-185. 
L'ambition collective et assumée de fonder une science de la règle électorale à l'orée du siècle échoue moins à cause des faiblesses académiques de la doctrine constitutionnelle sur certains points (le confinement de la production doctrinale au niveau doctoral, le peu d'originalité dans le choix récurrent des mêmes sujets de thèse, la dépendance du droit comparé à des sources de seconde main, la confusion littérature savante/militante, etc.) mais bien plutôt en raison « des cécités propres à l'objectivation de ce point de vue particulier ${ }^{73}$ qu'est le droit public positif de ces années qui, trop occupé à comprendre les institutions encore incertaines établies en 1875 , ne voit pas que la plupart des critiques qu'il professe sont déjà, de facto, intégrées dans les débats publics tant au sein de la sphère politique que dans les cénacles élargis qui entourent cette dernière. Le mélange des genres entre l'intérêt militant (reposant souvent sur la revendication de la représentation proportionnelle) et l'investissement intellectuel est par exemple tout à fait visible dans cette lettreproposition qu'un étudiant pour le doctorat, qui réalise pourtant une thèse sur tout autre chose, adresse au député de sa circonscription qui se trouve être le rapporteur de la Commission du suffrage universel sur ces sujets ${ }^{74}$. Dans un exposé manuscrit de plusieurs pages, il affirme : "Je n'ai pas la prétention d'ailleurs d'éditer un système absolument nouveau ; je reconnais qu'il comprend des emprunts à différents systèmes déjà en vigueur, notamment aux systèmes suisses, mais je crois qu'il ne serait copié sur aucun d'eux. (...) Si cette proposition vous intéressant, il vous était agréable de me communiquer les objections dont elle peut être susceptible, je me mets avec plaisir à votre disposition pour les étudier $\rangle^{75}$.

En d'autres termes, l'avenir politique désiré ou repoussé dans ces écrits, sous la forme par exemple d'un scrutin législatif plus collectif et plus équitable, n'est que la somme des aspirations différentielles de ces membres de l'élite à s'investir dans les affaires de l'État ${ }^{76}$. Malgré l'utilisation de méthodes relativement nouvelles (études de débats parlementaires, études de presse) le droit constitutionnel n'a pas pu briser, sur cette question des modes de scrutin, l'isolement disciplinaire dont on sait qu'il a été au cours du $\mathrm{xx}^{\mathrm{e}}$ siècle un frein puissant à l'innovation dans les sciences sociales ${ }^{77}$. Le stade final du cursus universitaire favorise une telle configuration en expliquant grandement le caractère relativement peu innovant de cette connaissance des règles électorales qui échappera de ce fait - mais également pour d'autres raisons liées à la politisation de la question jusque dans les années 1950 - aux juristes et ceci principalement au profit des spécialistes de science politique quand bien même ils seraient issus académiquement de la communauté des juristes.

\footnotetext{
${ }^{73}$ LACROIX, 2005, 23.

${ }^{74}$ Le député en question n'est autre qu'Étienne Flandin... père de Pierre-Etienne qui soutient en 1914 sa thèse sur... la RP en Angleterre (thèse $n^{\circ} 19$ ).

${ }^{75}$ Lettre de B. Régis à E. Flandin, 31 octobre 1905 (Archives Nationales, 423 AP 4).

${ }^{76}$ Les fiches biographiques de la faculté de droit de Paris conservées aux archives nationales sont trop pauvres pour aller au-delà de la formulation de cette simple hypothèse hormis, bien entendu, les cas plus documentés de Menthon et Flandin.

${ }^{77}$ Dogan et Pahre, 1991.
} 
Le meilleur exemple est sans doute celui de M. Duverger dont on connaît l'apport essentiel à l'étude des modes de scrutin $^{78}$. Paradoxalement, M. Duverger choisit son sujet de thèse sans aucune anticipation de ce que seront ces terrains d'investigation intellectuelle : "Avec Bonnard, nous avions délimité un sujet de thèse d'une austérité parfaite : "L'affectation des immeubles domaniaux aux services publics ». Rien ne pouvait faire plus sérieux : les universitaires ayant tendance (...) à confondre le sérieux et l'ennuyeux $\gg{ }^{79}$. Les conditions du choix des thèses sur la représentation proportionnelle ne sont pas du même ordre : moins tenues alors par des exigences strictement académiques elles ressortent d'un intérêt indirect pour la politique professionnelle qui est alors en train d'advenir et qui génère dans les franges intellectuelles de la moyenne bourgeoisie de la Belle Époque une inflation d'activités de commentaires électoraux ${ }^{80}$. Mais en tant que savoir concédé par le corps professoral, cette contribution doctorale au marché du commentaire électoral est condamnée car ne possédant ni influence sur le moment ni postérité dans l'historiographie doctrinale. Ce faisant, la configuration ainsi décrite n'est sans doute pas sans effet sur la faiblesse plus générale et plus récurrente, en France, des savoirs constitutionnels en matière de connaissance des modes de scrutin.

L'abandon d'une telle réforme électorale du mode de scrutin législatif dans les projets de réforme institutionnelle de 1993 ou $2008^{81}$ s'explique du point de vue académique par le fait qu'elle était seulement portée par une minorité de juristes, professeurs et/ou participant aux instances d'État (Conseil constitutionnel, comités de réflexion, etc.), et non par l'ensemble d'une communauté disciplinaire qui a depuis longtemps délaissé ce terrain d'étude à d'autres.

Thomas MARTY

GAP - Université Paris Ouest GSPE - Université de Strasbourg thomarty@hotmail.com

\footnotetext{
${ }^{78}$ DuVErger, 1951.

${ }^{79}$ Duverger, 1977, 74.

${ }^{80}$ Marty et SchWartz, 2008, 79-105.

${ }^{81}$ Pauvert, 2007.
} 


\section{Sources}

Sont rassemblées ici les références intellectuelles juridiques mobilisées dans la controverse doctrinale, notamment à travers les jurys de thèse (docteurs eux-mêmes, membres des jurys) : quelques-unes des thèses bien sûr, essais critiques sur le système électoral publiés par les professeurs de droit principalement, supports de cours diffusés commercialement (traités, manuels) ou non (cours tapuscrits, "répétitions écrites »), articles et comptes rendus des thèses elles-mêmes. Quelques ouvrages plus nettement militants (Lachapelle, La Chesnais), lus par les juristes, viennent compléter cette liste.

Académie de Toulouse., 1885, Programme des cours et conférences de l'enseignement supérieur, Toulouse, A. Chauvin, 1885 (Archives Nationales, F/17 13155-13156).

Archives Nationales, 423 AP 4, dossier 4 : correspondance 1893-1909. Archives privées Étienne Flandin.

Archives nationales, F/17/13141-13156. Programmes imprimés des cours et conférences d'enseignement supérieur (annuaire des facultés, livrets de l'étudiant), classés par académies : $1883-1898$.

Archives nationales, AJ 1628/AJ 1630/AJ 1645/AJ 1674/AJ 1676. Académie de Paris, faculté de droit 1805-1952, fiches individuelles de scolarité et d'inscription, vers 1815-1920.

BARTHÉLÉMY J., 1912, L'organisation du suffrage et l'expérience belge, Paris, M. Giard et E. Brière.

BARthélÉMy J., 1918, Le problème de la compétence dans la démocratie, Paris, Alcan.

BARThÉlÉMY J., 1928, La crise de la démocratie représentative, Paris, Giard.

BARThÉlÉmy J., 1931, La crise de la démocratie contemporaine, Paris, Sirey.

BAtBiE A., 1861, Introduction générale au droit public et administratif, Paris, Cotillon.

BESSON A., 1897, Essai sur la représentation proportionnelle de la majorité et des minorités, Dijon, Jobard.

Blavet J., 1923, Le scrutin de liste et la représentation proportionnelle dans la loi du 12 Juillet 1919, Alès, Clarapède.

Cameau P., 1901, La représentation proportionnelle en Belgique, Paris, A. Rousseau.

CAmpagnole E., 1885, La démocratie représentative : représentation proportionnelle de la majorité et des minorités, Paris, F. Pichon.

Chardon J.-L., 1910, La réforme électorale en France, Paris, Arthur Rousseau.

DuBoIs P., 1906, La représentation proportionnelle soumise à l'expérience belge, Lille, Imprimerie H. Morel.

Duguit L., 1928, Traité de droit constitutionnel, Tome 2, Paris, E. de Boccard, $3^{\mathrm{e}}$ édition, $726-753$.

Duthoiт E., 1904, La représentation proportionnelle. Les leçons de l'expérience belge 1900-1904, Lille, Imprimerie H. Morel, 1904

Duverger M., 1951, Les partis politiques, Paris, Armand Colin.

Duverger M., 1977, L'autre côté des choses, Paris, Albin Michel.

EsmeIn A., 2001, Éléments de droit constitutionnel français et comparé, Paris, Édition Panthéon Assas, rééd. 
Exposition internationale universelle., 1905, Congrès international de Droit comparé tenu à Paris du 31 juillet au 4 août 1900, Paris, LGDJ.

FonTAINE J., 1909, La représentation proportionnelle en Wurtemberg, Paris, Larose et Tenin.

Galland J.-J., s.d, Notre système électoral et la représentation proportionnelle, Grenoble, L. Aubert.

La Chesnais P. G., 1902 La Représentation proportionnelle et les partis politiques, Paris, Société nouvelle de librairie et d'édition.

Geouffre de Lapradelle A., 1912, Cours de droit constitutionnel, Paris, Pedone.

Gidel G., 1938-1939, Répétitions écrites de droit constitutionnel comparé, Doctorat, Paris, Les cours de Droit.

JAIGU G., 1911, La représentation proportionnelle, Rennes, Impr. des arts et manufactures.

Lachapelle G., 1911, La Représentation proportionnelle en France et en Belgique, Paris, Félix Alcan.

Le Fur L., 1934, La démocratie et la crise de l'État, Archives de la philosophie du droit et de la sociologie politique, Cahiers 3-4, 7-49.

de Menthon F., 1946, Vers la IVE République, Paris, Hachette.

Mestre A., 1899, Le fondement juridique de l'élection proportionnelle, Revue Générale du Droit, Tome XXIII, 444- 464

Mestre A., 1928, Etudes et étudiants (chroniques du Figaro), Paris, Librairie Dalloz.

Petitjean T., 1915, La représentation proportionnelle devant les chambres françaises. Étude d'histoire parlementaire et législative, Paris, Sirey.

Le proportionnaliste, $1906, \mathrm{n}^{\circ} 6,1^{\mathrm{er}}$ octobre.

Saleilles R., 1898, La représentation proportionnelle, Revue du droit public et de la science politique, Tome 9, 215-234 et 385-414.

SAleilles R., 1899, Compte rendu de la thèse de N. Saripolos, Nouvelle revue historique du droit français et étranger, Tome 23, 591-604.

SARIPOlos N., 1899, La démocratie et l'élection proportionnelle, Paris, Arthur Rousseau.

Vernes M. (dir.), 1888, Société pour l'Étude de la Représentation Proportionnelle. La représentation proportionnelle : études de législation et de statistique comparées, Paris, Pichon.

VILlEy E., 1900, Législation électorale comparée des principaux pays d'Europe, Paris, Larose.

Villey E., 1910, Les périls de la démocratie française, Paris, Plon.

Villey E., 1918, Les vices de la constitution française, Paris, Plon-Nourrit et Cie. 


\section{Bibliographie}

Aromatorio S., 2007, L'absence traditionnelle du mode de scrutin dans la constitution française : cause de l'instabilité chronique des modes de scrutin législatifs, Revue française de droit constitutionnel, 71, 601-622.

Bourdieu P., 2001, Science de la science et réflexivité, Paris, Editions Raisons d'agir.

Bigot G., 2007, Larnaude (Ferdinand), in Arabeyre P., Halpérin J.-L. et Krynen J. (dirs.), Dictionnaire historique des juristes français, $X I I^{e}-X X^{e}$, Paris, PUF, 466 $-467$.

Burney J.-M., 1988, Toulouse et son université, Facultés et étudiants dans la France provinciale $d u X I X^{e}$ siècle, Presses du Mirail, éditions du CNRS, Toulouse-Paris, 133-146.

Charle C., 2004, Enseignement supérieur et expansion internationale (1870-1930) : des instituts pour un nouvel empire ? in Heilbron J, Lenoir R, Sapiro G, Pargamin P (dirs)., Pour une histoire des sciences sociales, Paris, Fayard, 323-347.

Charle C., 1994, La république des universitaires, Paris, Seuil.

Déloye Y et Ihl O., 2008, L'acte de vote, Paris, Presses de Sc. Po.

Dogan M. et Pahre R., 1991, L'innovation dans les sciences sociales : la marginalité créatrice, Paris, PUF.

Ducerf L., 2006, François de Menthon. Un catholique au service de la République (1900 - 1984), Paris, Cerf.

Favre P., 1989, Naissances de la science politique, Paris, Fayard.

Garrigou A., 2002, Histoire sociale du suffrage universel (1848-2000), Paris, Seuil.

Godechot O et Mariot N., 2004, Les deux formes du capital social. Structures relationnelles des jurys de thèses et recrutement en science politique, Revue française de sociologie, vol 45, $\mathrm{n}^{\circ} 2,35-74$.

Hakim N., 2007, Bufnoir C., in Arabeyre P., Op. cit., 143-145

Halpérin J.-L. b, Molinier V., in Arabeyre P, Op. cit., 568-569.

ImBert J., 1984, Passé, présent et avenir du doctorat en droit en France, Annales d'histoire des facultés de droit, I, 11-35.

KARADY V., 1986, Les universités de la troisième République, in Verger J (dir.), Histoire des universités en France, Toulouse, Privat.

Lacroix B., 1985, Conclusion, in d'Arcy F., La représentation, Paris, Economica, 175185.

LACroix B., 2005, Introduction : quelques observations avant de dire droit, in IsRAEL L., Sacriste G., Vauchez A., Willemez L. (dirs), Sur la portée sociale du droit, Paris, PUF, 19 -27.

Marty T. et Schwartz A., 2008, A bonne distance. Activités intellectuelles, expériences politiques et figures de l' « électeur profane » (fin $\mathrm{XIX}^{\mathrm{e}}$-début $\left.\mathrm{XX}^{\mathrm{e}}\right)$, in FrOMENTIN T. et Wолсік S., Le profane en politique : compétences et engagements du citoyen, Paris, L'Harmattan, 79-105.

MARTy T., 2010, Les réformes électorales législatives comme facteurs de sortie de crise (1875 - 1975), in GRÉvy J., Sortir de crise. Les mécanismes de résolution de crises politiques (XVI $-X X^{e}$ siècle), Rennes PUR, 109-122. 
Milet M., 2000, Les professeurs de droit citoyens. Entre ordre juridique et espace public. Contribution à l'étude des interactions entre les débats et les engagements des juristes français (1914 - 1995), thèse, science politique, Paris II - Assas.

Milet M., 2007, Le Fur (Louis) », in Arabeyre P., Op. cit., 485-486.

Moulinier P., 2001, Naissance de l'étudiant moderne, Paris, PUF.

NorRiel G., 1991, Les jugements des pairs. La soutenance de thèse au tournant du siècle, Genèses, n $5,132-147$.

Pauvert B., 2006, Élections et modes de scrutin, Paris, L'Harmattan.

PAuvert B., 2007, Les modes de scrutin dans les propositions du comité Balladur, Revue politique et parlementaire, vol 109, $\mathrm{n}^{\circ} 1045,83-88$.

Redor M.J., 1992, De l'État légal à l'État de droit. L'évolution des conceptions de la doctrine publiciste française, 1879-1914, Paris, Economica, 111-114.

SACRISTE G., 2001, Droit, histoire et politique en 1900. Sur quelques implications politiques de la méthode du droit constitutionnel à la fin du XIX ${ }^{\mathrm{e}}$ siècle, Revue d'histoire des sciences humaines, $\mathrm{n}^{\circ}$ 4, 69-94.

SACRISTE G., 2002, Le droit de la République (1870-1914) : légitimation(s) de l'État et Construction du rôle de professeur de droit constitutionnel au début de la Troisième République, Thèse, science politique, Paris I, 2 tomes.

SAUlnier F., 1996, Joseph Barthélémy 1874-1945. la crise du constitutionnalisme libéral, thèse, droit, Paris II.

SusANi N., 2007, Une aporie de la justice constitutionnelle française : l'impuissance du Conseil constitutionnel face à l'inconstitutionnalité de l'actuel découpage électoral par les élections législatives de 2007, Revue française de droit constitutionnel, 69, 145-159.

WALLON-LeduCQ C.M., 2004, Les fonctions et les usages politiques de la règle électorale (sous le regard, un siècle plus tôt, de Raymond Saleilles), in LAURENT A., Delfosse P., Frognier A. P. (dirs), Les systèmes électoraux : permanences et innovations, Paris, L'Harmattan, 293-307. 